\title{
Treading lightly on shifting ground: The direction and motivation of future geological research
}

\author{
Computer Consultant II, North Carolina Geological Survey, 2090 US Highway 70, Swannanoa, NC 28778, USA. E-mail: anne.witt@ncdenr.gov
}

The future of the geosciences and geological research will involve complex scientific challenges, primarily concerning global and regional environmental issues, in the next 20-30 years. It is quite reasonable to suspect, based on current political and socioeconomic events, that young geoscientists will be faced with and involved in helping to resolve some well defined problems: water and energy security, the effects of anthropogenic climate change, coastal sea level rise and development, and the mitigation of geohazards. It is how we choose to approach these challenges that will define our future. Interdisciplinary applied research, improved modeling and prediction augmented with faster and more sophisticated computing, and a greater role in creating and guiding public policy, will help us achieve our goals of a cleaner and safer Earth environment in the next 30 years. In the far future, even grander possibilities for eliminating the risk of certain geohazards and finding sustainable solutions to our energy needs can be envisioned. Looking deeper into the future, the possibilities for geoscience research push the limits of the imagination.

\section{Introduction}

"Any sufficiently advanced technology is indistinguishable from magic." - (Clarke, 1973)

In the 1960s and 1970s, British science fiction author and scientist Arthur C. Clarke proposed three 'laws'. These adages describe our modern interpretation of advanced technology by future civilizations as seen through the context of our current technological limitations. The third law (above), written as an accompaniment to his 1973 revised collection of essays, 'Profiles of the Future: An inquiry into the limits of the possible', is probably his most well known. There is no reason to suspect that technologies, which we may consider to be impossible or even laughable now, may be a reality in the future. Our lofty goals of predicting the timing of volcanic eruptions and earthquakes, controlling ocean circulation and weather patterns, are all imagined possibilities that modern scientists can only dream about and continue working towards. I see no place in the far future without the help and expertise of various geoscientists; on this planet, or elsewhere in the solar system. However, in order to forecast a realistic conjecture of a far future world shaped by advances in the geosciences, it is important to recognize the variables that will affect our more immediate future. One cannot step so far forward, without first looking backwards at where we have been.

The first Geological Survey in the United States was started in 1823 in North Carolina through a legislative order by the North Carolina General Assembly. The sole scientist in charge of the Survey, Denison Olmsted, was instructed to find and map sources of mineral and rock material that would benefit the state monetarily, and elucidate the geological resources of North Carolina for the greater good of the public. His prime objectives were to find material to promote agribusiness and identify precious metals, including gold. The first geological map of the state, completed and delivered to the Department of Agriculture in 1825, was influential in directing the service of other geological surveys to follow. In the nearly 200 years since the completion of this map, little has changed. Many geologists and geoscientists are still heavily involved in the identification and retrieval of natural resources, although the scope of what is now considered "precious" has expanded to include such intrinsic concepts as clean air and water. The notion of what a geoscientist does in the workplace is also expanding and merging with other scientific disciplines. The momentum of the "green economy" has required that recent graduates have knowledge in many disciplines (meteorology, geology, chemistry, biology, etc.) and be able to integrate with other fields of study to solve modern problems. These problems not only present great challenges, but also provide exciting and relevant avenues for new research.

\section{Future research trends in the geosciences}

For the past 50 years, geologists have been employed in such endeavors as traditional geologic field mapping, mining and the petroleum industry, and more recently, hydrology and environmental fields. Earth science research has generally focused solely on understanding and visualizing the interaction of the Earth's systems. The effects of anthropogenic forcing have now reached critical levels where natural processes are being disrupted. Human interference in our natural systems can no longer be excluded from geological research. Potential future research trends will involve issues related to the climate, natural resources and geohazards, primarily driven by the needs of an ever increasing global population. Galloway (2010) postulated that future geosciences research, and subsequent funding, will be in three general areas: cross-disciplinary science, modeling and prediction, and scientific communication to society. I would also insert into this list, that we will see an institutional shift from "science for the sake of science" to applied science for the benefit of humanity. 
The increased interest in the interdisciplinary nature of science is primarily due to a push within the physical sciences over the past 20 years to promote the concept of the Earth as a system. This concept focuses on the interconnectedness and interaction of the Earth's individual parts (e.g. the biosphere, lithosphere, atmosphere, etc). The emerging concept of "Earth system science" will redefine geoscience research in the $21^{\text {st }}$ century and the tasks we will be asked to accomplish. No longer can the natural sciences function in a vacuum. There will be a fundamental shift away from study within an individual discipline to interdisciplinary sciences, as hiring in government, academic and private industry will require a more diverse workforce. There will also be a need to incorporate and work with our colleagues in the social and economic sciences. By breaking down barriers between the individual disciplines we can improve modeling and prediction on the global scale, within the Earth System.

There will also be more geoscientists working on applied scientific problems to creatively provide applied solutions to global and regional dilemmas. Probably our most pressing challenge will be serving the world's growing energy demands. Our need for fossil fuels certainly will dominate the socioeconomic and political stage in the immediate future, and perhaps into the far future (without a viable and economical energy solution). Demands will also increase on our other natural resources including available fresh water in our river and aquifer systems. The availability of clean, potable water is already a challenge in many developing countries and will continue to require more attention as world population grows. Future research efforts will need to focus on providing long-term water security and more robust modeling of fresh water occurrence, distribution, and sustainability.

The effect of human interaction and modification of our coastal areas will become a major concern in our immediate future. Near shore systems are particularly vulnerable to changes in local sea level rise further exacerbated by the specter of anthropogenic climate change. Even though the world's coastlines only occupy a small percentage of the world's habitable land, they represent the part of the ocean system that humans actively use and benefit from economically. The continued increase in population in coastal areas will require applied solutions to mitigate potential damage from overuse and protect offshore and near shore ecosystems.

As human populations continue to push to farther corners of the world, they will also encounter greater geologic hazards. More people are living in active seismic zones, nearer to volcanoes, and on steeper slopes in landslide prone areas. Further research into the earth's interior will help provide insight into the mechanisms behind earthquake generation and a more systematic approach to earthquake prediction. Increased instrumentation, qualitative observation and predictive modeling will improve our understanding of the timing of volcanic eruption and improve the modeling of landslide hazards.

\section{Advanced technology: Improvements in modeling and prediction}

Many of these global problems will require advances in probabilistic modeling and prediction at various temporal and spatial scales. It is to our benefit that young geoscientists have been primed to approach some of these multi-faceted challenges and are particularly adept at utilizing technology. Never before has a generation had such computing power available, in such near real-time, at any other time in our history. Since the 1960's, science students have been inundated with environmental dogma, learning of the unintended human-induced effects on our ecosystems. More recently, young scientists have matured in a world with the personal computer and the Internet, and are uniquely skilled in comprehending and utilizing this technology. These individuals live in a wired world, interacting together as a global community. While technological innovation has been changing at a blinding pace, our computing ability has also grown exponentially in the past 20 years. Smaller and more mobile data storage and faster processing speeds have made technology more widely available at lower prices. As long as the Internet is available cheaply and without restriction, global datasets can be made accessible for interpretation and application to myriad problems.

A discussion of the future of the geosciences cannot take place without mentioning the advances in GIS technology. It was not long ago that geospatial platforms were only used by specialists toiling away in the production of 2-D maps and CAD drawings. More sophisticated software is allowing for earth visualization in 3-D and 4-D. Mobile hand-held devices such as smart phones, laptop PCs, and even GPS navigation systems in cars, have placed geospatial data in the hands of users who need no special training. Thanks to programs like 'Google Earth,' aerial photography and digital topographic data at varying scales are now available to nearly everyone for interpretation. This seemingly simple program is being utilized by numerous organizations for a variety of uses, from the academic to the mundane.

The availability and inherent usability of 'Google Earth', and other open-source geospatial programs, is one that the scientific community can use as a model for future data dissemination. Various agencies and universities worldwide are collecting massive amounts of physical observational data and storing these away, waiting for a time when these observations can be utilized. The challenge for the geoscience community is to bring datasets from these various disciplines together, in a rational way, rather than have distinct, separate communities working in isolation. Instead, cross-disciplinary work can connect scientists together to create cohesive observation networks for modeling and prediction purposes.

Comprehensive data networks will allow for modeling at ever greater temporal and spatial scales. These datasets should be expandable to the global scale, but must also be able to address and identify patterns for modeling in regional and local areas. While anthropogenic climate change is certainly a global phenomenon, the effects of changes in weather patterns and circulation are being recognized regionally. There also must be significant improvement in accurately modeling the effects of localized but devastating megaevents such as hurricanes, floods or large earthquakes. Temporally, these improved datasets will allow us to model natural systems far into the geologic past, in order to discern patterns that will help predict outcomes far into the future.

At the same time, scientists will need ever larger datasets at greater resolution to produce more realistic and complex models with greater model accuracy. These immense datasets will require expertise that is not usually taught as part of the core earth science curriculum. Complex data visualization, data mining and assimilation, and database management will involve collaboration with our colleagues in statistics, mathematics and computer programming. Since new models are often data driven, new methods of collecting these data will need to be created. Where remote sensing is not realistic or possible, new monitoring systems will be developed and our data array expanded to provide a more realistic framework for complex natural system modeling. 
Creating these interdisciplinary networks within the open framework of "cloud computing" will provide easier collaboration and data dissemination by allowing data and maps to be collectively stored on the Internet. This will eliminate the need for static data storage, costly infrastructure, and onsite network management. Once the dataset is made available, various users can then access these data for a variety of means and uses. The advancement of "cloud computing" and GIS-based web mapping will have a profound benefit for developing and emerging countries that may not have the capital to invest in data centers.

Unfortunately, some technological advances may make more traditional geologic endeavors, such as field mapping, obsolete. In the future, these tasks will continue to move out of the field and into the office. Already, smaller field laptops have begun to replace field notebooks and better GPS location devices have significantly improved our mapping techniques. Digital topographic imaging using LiDAR has proven successful for identifying major lineaments and fracture systems. Smaller projects are using mounted high-resolution LiDAR devices to scan and continuously monitor slow moving rockslides and displacement along faults. Continued advances in remote sensing and smaller, hand-held spectrometers for mineral and rock identification will decrease the field time needed for long-term mapping projects. In the immediate future, there will still be a place for having "boots on the ground" to collect samples and complete detailed investigations for individual projects, but field time will be much more efficient and limited.

\section{Communicating geoscience: Science for the greater good}

Communicating complex scientific problems to a variety of audiences will also be a great challenge for geoscientists. Educating the public and decision makers will be necessary to provide popular momentum to fund and support further geoscience research and to encourage young people to enter the field. There will need to be a continuous effort to train geoscientists in effective communication and appropriate ways of interfacing with local communities and stakeholders. Complex geological ideas will need to be described in a way that is scientifically accurate, complete and understandable to diverse populations with a variety of skill levels and little scientific background.

It is also reasonable to suspect that geoscientists, based on our inherent knowledge and experience with environmental issues, will become even more involved in political decisions by helping to create and write public policy. The geoscience community will also have increasing international visibility and will play an important role in reinforcing the capacity of developing countries to mitigate and resolve environmental and natural resource issues. To that end, there will be an ongoing need to hire and collaborate with geoscientists with an expertise in business management, political science, language and communication skills, and even human psychology.

Perhaps one of the greatest society benefits that future geoscientists can provide is the protection of life and property during natural disasters and the improvement of early warning systems. In crisis situations, the geologic community will play a critical role in providing concise geologic information to decision makers for emergency management decisions. This will involve moving beyond the era of the static geohazard map and even beyond real-time forecasting of individual hazards, such as a heavy rain event. A system will need to be put in place that can predict specific dangerous weather patterns, within several hours to days, with sufficient lead time for action by those directly impacted. This will mean being able to identify discrete areas affected by specific hazards before the event unfolds. Individual home owners can be systematically warned of flooding and landslide dangers with specific instructions of what to do. Emergency personnel can be directed, safely, where they will be needed and most efficiently utilized. Whatever level of predictive skills and techniques that we develop, it will be imperative for geoscientists to be able to educate, communicate and motivate the public to understand that the events that are forecasted are real and imminent, so as to limit potential loss of human life.

\section{Geological imagining: The far future of the geological sciences}

Oddly, there have been very few writers of speculative fiction who have delved into the idea of what geologic exploration and innovation will be like in the future, 100 or even 1000 years from now. This seems strange as there have been geologists sent to the moon, completed research on the international space station and others who are currently working on the Mars missions. There have been a few stories dealing with the mining of raw materials in space, mainly involving asteroids or distant planets. It seems even stranger when one realizes how instrumental the geosciences will be in shaping our future world. Indeed, if one of our goals as a civilization is to move beyond this planet and further into the universe, comparative planetology and planet exploration will be a critical function of the future geoscientist. How is it that the geosciences have been reduced to the background players in stories where we should be leading characters?

Perhaps the reason is because we have not yet made the scientific discoveries that will quantify and solidify what seem like geological imaginings. Some of these are not steps along the road to discovery, but leaps from where we are today. They will require continued long-term research, continual funding availability, and extensive data collection. In the future, geoscientists will make systematic and accurate earthquake and volcanic eruption prediction a reality. Geoscientists will be able to better manage and modify the behavior of the world's oceans, weather patterns and global temperature for our benefit. Long-term and accurate modeling of landslides, tsunamis and other natural hazards will eliminate loss of life and property.

In the far future I am also hopeful that we will be able to move away from nuclear fission and harness nuclear fusion as an alternative energy source. However, our civilization will also require raw materials. Without a viable way to use our mineral resources with optimum efficiently or create them artificially, it is reasonable to assume that we will eventually be forced to look off our planet for raw materials. Some have speculated that we will be able to capture and tether an asteroid in high orbit to mine for metals. Further advancement in the fields of nanotechnology and robotics will also help us explore places formerly unavailable to humans: the interior of the earth, the deep ocean, and deep space.

Our goals are lofty, but generally seem to revolve around the control of our fate as a species on this planet. Ultimately, the role of geoscientists will be to control and modify our environment to make it more livable, to provide the raw materials and energy to power our civilization, and to make sustainable environs, wherever that may be. 


\section{Conclusion}

Young geoscientists have a particular stake in the future of the geological sciences and our world. Undoubtedly, unforeseen world events will change the outcomes of any prediction and reshape our influence on the future. We also cannot exclude the influence of political pressure on the outcomes of science by directly guiding funding to particular studies while "starving" others that are of less interest or intrinsic value. The current global economic recession is already stressing sources of funding for the geosciences. In reality, current funding levels will need to be maintained or significantly increased to help meet the realities of our changing world. We will need to be able to effectively communicate these critical issues to our political leaders, decision makers, and private industry.

With all of our technological advances and potential for multidisciplinary collaboration, I still foresee a need to provide young geoscientists with a strong background in the fundamentals of rudimentary science. One cannot understand the Earth System as a whole without first exploring and understanding how these individually subsystems operate. As a civilization matures, individuals specialize in disciplines in which they excel. Our new world will be made of scientists with specialties in their individual fields, working together with other specialists to bring together ideas and tackle new problems. For young geoscientists, these will be some of the greatest challenges and accomplishments of our generation.

\section{References}

Clarke, A.C., 1973, Profiles of the future: An inquiry into the limits of the possible, Harper \& Row, New York, NY, 256p.

Galloway, D.L., 2010, The complex future of hydrogeology, Hydrogeology Journal, v. 18(4), pp. 807-810.

\title{
CALL FOR PAPERS
}

Episodes is a quarterly science and news journal of the International Union of Geological Sciences (IUGS). It focuses on the publication of results of scientific research and other information addressing issues of interest to the global Earth science community. Special emphasis is given to topics involving geological aspects of population growth and economic development and their resulting impacts on or implications for society. As the principal publication of the IUGS, Episodes also carries information about IUGS scientific programs and activities to the extent necessary to communicate effectively with the worldwide IUGS constituency.

Contributions of the following types of manuscripts are solicited:

- Review papers

- News and views

- Information on training courses (especially those geared to participants from developing countries)

- Noteworthy new publications, including national or regional geological maps

Episodes also invites photos or other images for the front cover. Photos must be of high technical quality and tell an interesting geological story. A color transparency and one color print (at least $9 \mathrm{~cm} \times 12.6 \mathrm{~cm}$ ) are required for submission, which should be supplemented with a short explanatory paragraph (no more than 100 words).

Please address all contributions to:

\author{
Dr. M. Jayananda - Editor \\ Episodes \\ Geological Society of India \\ No.63, $12^{\text {th }}$ Cross, Basappa Layout \\ Gavipuram P.O., P.O. Box 1922 \\ Bangalore - 560 019, INDIA \\ Phone: +91-080-22422943; Telefax: +91-80-26613352 \\ Email: episodes.editor@gmail.com
}

